\title{
Lower Patient Anxiety and Unchanged Levels of Adherence to Hemato-Oncologic Treatment in Response to New Measures to Reduce Hospital Exposure Risk to COVID-19
}

\author{
Nadav Sarid ${ }^{1,2}$ \\ Shir Mann' \\ Yair Herishanu',2 \\ Chava Perry ${ }^{1,2}$ \\ Yael C Cohen ${ }^{1,2}$ \\ Inna Passage' \\ Miriam Neaman ${ }^{1,2}$ \\ Noam Benyamini ${ }^{1,2}$ \\ Maayan Jean' \\ Irit Avivi $\mathbb{D}^{1,2}$ \\ 'Departments of Hematology, Tel Aviv \\ Sourasky Medical Center, Tel Aviv, Israel; \\ ${ }^{2}$ Sackler Faculty of Medicine, Tel Aviv \\ University, Tel Aviv, Israel
}

\begin{abstract}
Objective: Hemato-oncology patients are at high risk for morbidity and mortality from coronavirus disease (COVID-19). The resultant heightened anxiety among these patients may negatively affect adherence to therapy and treatment-related outcome. We aimed to assess whether the adoption of precautionary measures provided by the medical team led to a reduction in COVID-19-related anxiety and, consequently, to successful execution of treatment plans.
\end{abstract}

Methods: All adult hemato-oncology patients actively treated or being followed-up at the outpatient service at Tel Aviv Sourasky Medical Center between March 25 and May 3, 2020, were invited to answer a questionnaire that focused on their anxiety and adherence to treatment following new measures to reduce risk of infection during the first COVID-19 outbreak.

Results: One hundred and fifty patients (representing 24\% of those being approached), average age 67 years, 52\% male, and 57\% undergoing antineoplastic therapy, responded to the survey. The introduction of precautionary measures resulted in a significant reduction in anxiety level in all patients, irrespective of age, sex, or treatment status. Attendance to scheduled visits in day care and outpatient clinics remained unchanged. Adherence to planned blood and imaging tests were $81 \%$ and $73 \%$, respectively, and $93 \%$ of the patients were satisfied with their medical care. Thirty-two percent of patients used telemedicine. Satisfaction with telemedicine was highest among non-actively treated patients and those experiencing high anxiety levels.

Conclusion: Reorganization of the hemato-oncology unit and provision of information to patients reduced COVID-19-related anxiety and enabled the same delivery of therapy as that prior to the pandemic.

Keywords: anxiety, treatment adherence, COVID-19

\section{Introduction}

The coronavirus disease (COVID-19) pandemic is caused by severe acute respiratory syndrome coronavirus 2 (SARS-CoV-2) and it continues to rage worldwide since its first emergence in China in December 2019. ${ }^{1}$ Despite the employment of home isolation of those diagnosed with or exposed to COVID-19 and the enforcement of social distancing, community-acquired infections are continuously expanding in Israel. $^{2}$ Older age, concomitant diagnosis of cancer, chronic cardiac,
Correspondence: Irit Avivi

Department of Hematology,

Tel Aviv Sourasky Medical Center,

6 Weizmann Street,

Tel Aviv, 6423906, Israel

Tel $+972-3-6973576$

Fax+972-3-6974452

Email iritavi@tlvmc.gov.il 
pulmonary, and kidney disease, obesity, smoking, and type 2 diabetes mellitus are all recognized as being associated with worse outcome and higher risk of death due to COVID-19..$^{3-5}$

Patients diagnosed with hematological malignancies, such as non-Hodgkin lymphoma (NHL), Hodgkin lymphoma (HL), multiple myeloma (MM), and chronic lymphocytic leukemia (CLL), often present with significant immunodeficiency and are, therefore, considered to be at higher risk of severe COVID-19. ${ }^{6-10}$ As such, patients diagnosed with hematological malignancies are often concerned about attending medical facilities, assuming that the risk of exposure to SARS-CoV-2 is greater than the risk associated with postponing their scheduled antineoplastic therapy. ${ }^{11}$

It is imperative to adopt a tailored management strategy in which patients that require active therapy will be reassured to continue their treatment providing that there are conditions in place that ensure their safety, whereas patients that completed therapy may be followed virtually by telemedicine.

The Hematology Division at the Tel Aviv Sourasky Medical Center (TASMC) implemented several measures aimed at reducing the risk of SARS-CoV-2 infection in non-hospitalized patients who are being treated for hemato-oncologic malignancies. The current study investigated patient anxiety and adherence to treatment in response to the safety measures employed to ensure a noninterrupted and safe antineoplastic treatment protocols during the first COVID-19 outbreak.

\section{Methods}

\section{Patient Population and Study Design}

Six-hundred and thirty patients aged $\geq 18$ years old and diagnosed with non-NHL, HL, CLL, and MM actively treated or followed in the outpatient services at the TASMC between March 25, and May 3, 2020 (the first COVID-19 outbreak in Israel) were invited to take part in the current non-interventional clinical study.

The study was approved by the Tel Aviv Sourasky Medical Center Institutional Review Board (Helsinki Committee), and was conducted in accordance with the Declaration of Helsinki. Patients answered a questionnaire in which they did not need to provide identifying information other than their age and gender. The questionnaire could not be cross-referenced with responders, ensuring their anonymity. Thus, we received an exemption from signing a consent form for participating in the study. All patients received a short message service (SMS) text and/ or an email inviting them to respond to a questionnaire that investigates their level of anxiety and adherence to treatment following the protective measurements newly implemented by the department with the aim of avoiding exposure to SARS-CoV-2 and ensuring safe uninterrupted provision of therapy during the first COVID-19 outbreak. The survey was sent out five weeks after the introduction of these preventive measures, first implemented at the end of March 2020.

\section{Protective Measurements Employed by the Department to Avoid Exposure to SARS-CoV-2}

1. Continuing communication with patients: Weekly newsletters were sent by SMS text and emails for providing updated recommendations and information. A dedicated mailbox and telephone line were provided, enabling patients to address their concerns about the COVID-19 outbreak.

2. Defining patients requiring "in-hospital visits" vs those that can be safely managed by "remote communication". 12

3. Ensuring safe visits in the clinic and day care facility: We established a pre-visit telephone interview in order to ensure the absence of any potential COVID-19-related symptoms that could potentially exposure the clinic attendees and staff to a COVID-19-infected patient. In addition, a separate in-hospital path was provided and patients were instructed to wear facemasks continuously and arrive unaccompanied when possible.

4. Isolation and referral to treatment of patients reporting symptoms potentially related to COVID-19.

\section{Study Questionnaire}

The questionnaire (presented in detail in the Supplement) included 7 sections (A-G):

(A) Patient characteristics (sex, age, education, socioeconomic data, diagnosis, treatment stage).

(B) General behavior (irrespective of hospital attendance) during the first COVID-19 lockout.

(C) Adherence to scheduled visits and treatment plan in day care and in the outpatient clinic prior and during the COVID-19 outbreak. 
(D) Anxiety level prior and after the adoption of new strategies to reduce exposure to SARS-CoV-2. Patients were asked to rate their anxiety of attending the hospital, using a six-point Likert scale questionnaire, ranging from "not concerned" to "highly concerned". Patients were asked to semiquantify their anxiety level, before and after protective measures were taken.

(E) Patient satisfaction with strategies employed to reduce the risk of infection.

(F) Patient satisfaction with the adoption of telemedicine.

(G) Patient perspective on the impact of COVID-19 outbreak on the quality of treatment.

\section{Statistics}

Categorical variables were reported as frequency and percentage. Continuous and ordinal variables were reported as mean and standard deviation or median and interquartile range (IQR). Categorical variables were compared between the two time points with the McNemar test, while continuous and ordinal variables were compared with the Wilcoxon signed-rank test. The Mann-Whitney test was applied to compare continuous and ordinal variables between groups of patients. All statistical tests were two-sided, and $\mathrm{p}<0.05$ was considered statistically significant. SPSS software was used for all statistical analyses (IBM SPSS Statistics for Windows, version 25, IBM corp., Armonk, NY, USA, 2015).

\section{Results}

\section{Patient Characteristics}

One-hundred and fifty patients (representing 24\% of those who received the link for the questionnaire), 78 (52\%) males, median age 67 years (IQR 55-73), responded to the survey. Seventy-three percent $(n=109)$ were married, and their median number of children was 2 (IQR 2.0-3.0). Eighty-four percent $(n=124)$ had a high school/college education. Fifty-nine percent $(n=89)$ were diagnosed with lymphoproliferative disease (including NHL, HL, and CLL), 33\% ( $\mathrm{n}=49)$ with MM, and $8 \%$ $(n=12)$ with leukemia. Fifty-seven percent $(n=86)$ were receiving an antineoplastic treatment at the time of this survey: $44 \%(n=66)$ were treated in the outpatient clinic and $13 \%(n=20)$ were treated at home solely by tablets (Table 1).
Table I Patient Characteristics $(n=\mid 50)$

\begin{tabular}{|l|c|}
\hline Age (Years), Median (IQR) & $67(55-73)$ \\
\hline Male, $\mathrm{n}(\%)$ & $78(52 \%)$ \\
\hline Family status, Married, $\mathrm{n}(\%)$ & $109(73 \%)$ \\
\hline Number of children, median (IQR) & $2(2.0-3.0)$ \\
\hline Rooms per person, median (IQR) & $0.5(0.4-0.75)$ \\
\hline High school/college education & $124(84 \%)$ \\
\hline Hematological diagnosis & \\
Lymphoproliferative disease, $\mathrm{n}(\%)$ & $89(59 \%)$ \\
Multiple myeloma, $\mathrm{n}(\%)$ & $12(33 \%)$ \\
Leukemia, $\mathrm{n}(\%)$ & $94(63 \%)$ \\
\hline $\begin{array}{c}\text { Time from diagnosis } \\
<5 \text { years, } \mathrm{n} \text { (\%) }\end{array}$ \\
\hline
\end{tabular}

Abbreviation: IQR, interquartile range.

\section{General Understanding and Adjusted Habits During the First Corona Outbreak} Sixty-eight percent $(\mathrm{n}=103)$ of the responders considered COVID-19 to be more contagious than flu infection, and $91 \%(n=136)$ knew that SARS-CoV-2 spreads in airborne droplets. Ninety-four percent $(n=141)$ reported that they wear a mask whenever they leave home, and $93 \%(n=140)$ reported that they maintain social distancing. Ninety-five percent $(\mathrm{n}=142)$ of the responders completely avoid having visitors at home and $50 \%$ do not leave home except for essential (mostly medical) requirements.

\section{COVID-19-Related Anxiety Level Before and After the Introduction of Safety Measures (Figure I)}

Patients were asked to grade their level of anxiety from potentially being exposed to COVID-19 infection during their stay in the hospital as experienced before and after the adoption of the department's new risk-prevention measures. The mean anxiety score prior to the adoption of these new precautions was 4.25 (standard deviation [SD] 1.735). Females reported higher mean anxiety levels compared to males (4.61, SD 1.54 vs 3.92, SD 1.84, respectively, $\mathrm{p}=0.013$ ). Non-actively treated patients expressed higher anxiety levels when required to attend the hospital compared to actively treated patients $(4.67, \mathrm{SD} 1.73$ vs 4.02, SD 1.70, $\mathrm{p}=0.007$ ).

Age, education level, and type of the disease had no statistically significant impact upon anxiety level. The 


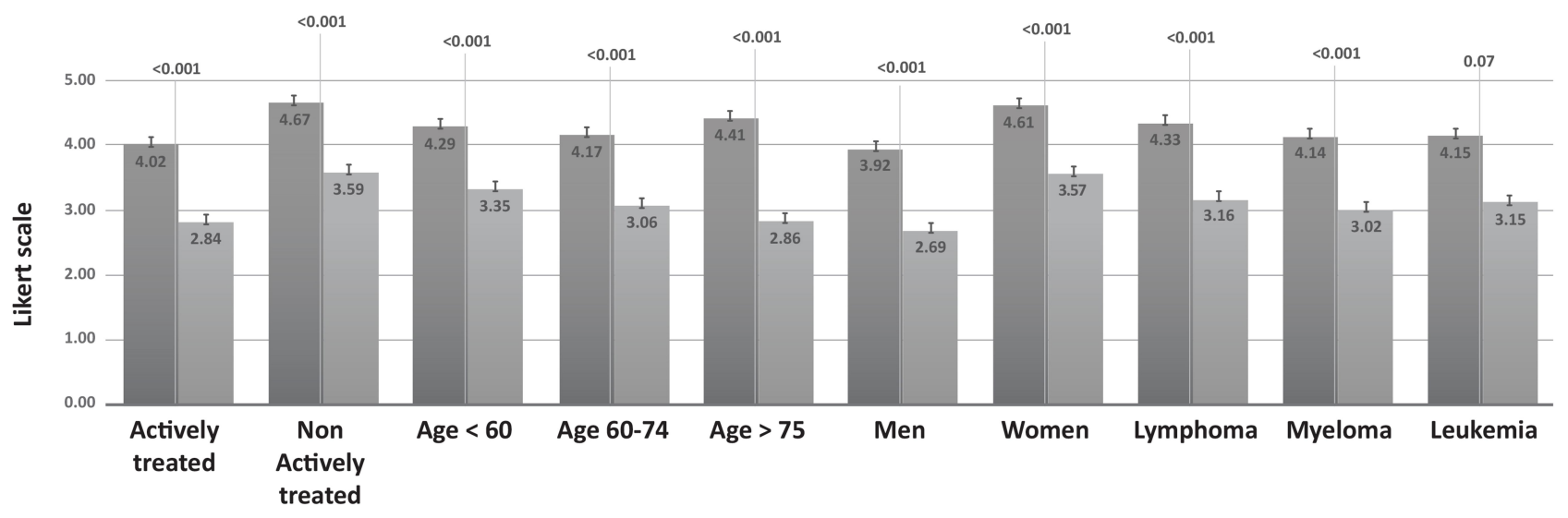

Figure I Anxiety level before and after the introduction of measures to reduce exposure to COVID-19 in the hospital.

adoption of new precautionary measures resulted in a significant reduction in anxiety levels to a mean of 3.11 (SD-1.645) and a median of 3.0 (IQR of 2.0-4.0) $(\mathrm{p}<0.001)$. The reduction in anxiety level was statistically significant among all patients, regardless of age, sex, and treatment status.

\section{Patient Satisfaction with Strategies Employed to Reduce Risk of Infection}

Seventy-seven percent of all patients $(n=116)$, of whom 76 were actively treated and 40 were non-actively treated, reported that they had received information about the new safety strategies before arriving to the clinic. Overall, $83.3 \%(n=125)$ were satisfied with the strategies that were implemented to reduce the risk of SARS-CoV-2 infection during their visit to the hospital. Table 2 presents the degree of satisfaction with the specific measurements. There were no statistically significant differences in the degree of satisfaction between actively and non-actively treated patients in all satisfaction-related parameters.

\section{Adherence to Scheduled Visits, Treatment Plan and Planned Medical Investigations}

There was no significant change in the frequency of visits of patients who were actively treated in the day care unit, with only $4.5 \%$ (3/66) of patients having reported COVID19-related delays in their scheduled treatment visits. The same applied to the patients attending the outpatient clinic, where $6 \%(9 / 150)$ only reported a delay in their planned

Table 2 Satisfaction with Strategies Employed to Reduce the Risk of Infection*

\begin{tabular}{|c|c|c|c|}
\hline Variable & $\begin{array}{c}\text { All } \\
\text { Patients }\end{array}$ & $\begin{array}{l}\text { Actively Treated } \\
\text { Patients }\end{array}$ & $\begin{array}{l}\text { Non-Actively Treated } \\
\text { Patients }\end{array}$ \\
\hline & $(n=150)$ & $(n=86)$ & $(n=64)$ \\
\hline $\begin{array}{l}\text { Direct arrival by elevator to hemato-oncology department, mean } \\
\text { (STD) }\end{array}$ & $5.17(1.305)$ & $5.01(1.448)$ & $5.50(0.878)$ \\
\hline Reducing the number of visitors, mean (STD) & $5.34(1.112)$ & $5.45(0.953)$ & $5.10(1.399)$ \\
\hline Use of protective measures by the medical team, mean (STD) & $5.45(0.978)$ & $5.39(1.043)$ & $5.57(0.815)$ \\
\hline Employment of telemedicine, mean (STD) & $5.15(1.286)$ & $4.98(1.377)$ & $5.33(1.168)$ \\
\hline Division to treatment capsules in day care unit, mean (STD) & $5.26(1.119)$ & $5.26(1.163)$ & NR \\
\hline Home treatment and home visit, mean (STD) & $4.92(1.570)$ & $4.66(1.758)$ & NR \\
\hline Outpatient "Drive In" treatment center, mean (STD) & $4.69(1.657)$ & $4.81(1.733)$ & NR \\
\hline
\end{tabular}

Notes: There were no statistically significant differences in the degree of satisfaction between aactively treated and non-treated patients in all parameters. *Likert scale questions: $0=$ Not relevant, I = No importance, 2 = Low importance, $3=$ Neutral, $4=$ Slightly important, $5=$ Important, $6=$ Very important.

Abbreviation: STD, standard deviation. 
Table 3 Adherence to Scheduled Visits, Treatment Plan and Planned Medical Investigations

\begin{tabular}{|l|c|c|c|}
\hline Variable & $\begin{array}{c}\text { All Patients } \\
(\mathbf{n = 1 5 0 )}\end{array}$ & $\begin{array}{c}\text { Receiving Active Treatment } \\
(\mathbf{n = 8 6})\end{array}$ & $\begin{array}{c}\text { Not Receiving Active Treatment } \\
(\mathbf{n}=64)\end{array}$ \\
\hline Avoided planned blood tests, $\mathrm{n}(\%)$. & $19 \%(29 / 150)$ & $18.6 \%(16 / 86)$ & $20.3 \%(13 / 64)$ \\
\hline Avoided planned imaging tests, $\mathrm{n}(\%)$. & $21 \%(31 / 150)$ & $15.1 \%(13 / 86)$ & $28.1 \%(18 / 64)$ \\
\hline Avoided medical appointments, $\mathrm{n}(\%)$. & $6 \%(9 / 150)$ & $4.6 \%(4 / 86)$ & $7.8 \%(5 / 64)$ \\
\hline
\end{tabular}

appointments (Table 3). Eighty-one percent $(n=121)$ of all patients underwent their scheduled blood tests. Nineteen percent $[\mathrm{n}=29 ; 13$ non-actively treated patients $(20 \%)$ and 16 actively treated patients $(19 \%)]$ reported that they chose to postpone their planned blood test, and most of them $(76 \%, \mathrm{n}=22)$ did not inform their treating physician about these delays. Seventy-three percent underwent their scheduled imaging tests. Thirty-one patients $(21 \%)$, of whom 18 were non-actively treated $(28 \%)$ and 13 were actively treated $(15 \%)$, chose to postpone their scheduled imaging tests, and $16(52 \%)$ did so without the approval of their treating physician (Table 3).

\section{Satisfaction of the Adoption of Telemedicine}

Forty-nine patients (32\%), of whom 23 (27\%) were actively treated and $26(41 \%)$ were non-actively treated used telemedicine for communicating with their physicians. Fortythree $(43 / 49,88 \%)$ of them found it useful compared with $71.3 \%(72 / 101)$ of those that did not use it $(\mathrm{p}=0.025)$. Patients who ranked virtual doctor appointments as a good solution had higher anxiety levels (mean 4.67, SD 1.60) compared to those who ranked it as a non-optimal solution (mean 3.94, SD 1.71, $\mathrm{p}=0.018$ ) or as a "disappointing" alternative (mean 3.91, SD 1.88, $\mathrm{p}=0.062$ ).

Sixty-one percent (30/49, 11 actively treated and 19 non-actively treated) of the patients that used telemedicine expressed their wish to continue with telemedicine visits after the COVID-19 outbreak as well, while only $32.7 \%$ $(33 / 101)$ of the patients were unwilling to use it $(\mathrm{p}<0.001)$. Patients who wished to continue virtual appointments had higher anxiety levels than those who did not (mean 3.48, SD 1.61 vs mean 2.85, SD 1.62, $\mathrm{p}=0.018$ ). Sixty-four percent of actively treated patients (56/87) expressed their wish to avoid telemedicine visits after the COVID19 outbreak vs $47 \%$ of non-actively treated patients (30/ 63) $(\mathrm{p}=0.041)$. Age, sex, education level, and time from diagnosis to answering the questionnaire did not influence patients' attitudes towards telemedicine.

\section{Patient Perspective on the Impact of the COVID-19 Outbreak on the Quality of}

\section{Treatment}

Eighty-one percent of the patients ( $\mathrm{n}=121)$, of whom $78 \%$ $(n=68)$ were in the actively treated group and $84 \%(n=53)$ were in the non-actively treated group, reported that the Corona pandemic did not affect the quality of medical care they received. Twelve percent, including $14 \%(n=12)$ in the actively treated group and $9 \%(\mathrm{n}=6)$ in the nonactively treated group, reported an improvement in the quality of their medical care. Only $6.66 \%(\mathrm{n}=10,6$ actively treated) reported a decline in the quality of medical care.

\section{Exposure to COVID-19 and Outcome}

During the study period, 4083 patient visits were recorded, with 1400 at the day care clinic and 2683 in the outpatient clinic (many of the patients had multiple visits).

There were no documented in-hospital exposures to COVID-19 infection during the entire study period.

\section{Discussion}

Patients diagnosed with hematologic malignancies are particularly at high risk for COVID-19-related complications and mortality, ${ }^{6-10}$ and are thus likely to express high anxiety levels when required to attend medical facilities for receiving their treatment. Here, we report the results of a survey on 150 lymphoma/MM patients, focusing on their COVID-19-related anxiety and its resultant impact on their adherence to treatment before and after the introduction of safety measures to reduce their risk for infection in the hospital.

COVID-19 carries with it significant medical and emotional morbidities, particularly in actively treated cancer patients who are known to be at higher risk for severe COVID-19 infection. ${ }^{13,14}$ As a result, those patients are often apprehensive about attending their medical appointments under the assumption that risk of exposure is especially high in medical centers. Avoiding exposure to 
medical services resulting in delayed diagnosis of cancer has already been shown to result in decreased overall survival. ${ }^{15}$ In line with that, avoidance or postponement of a planned antineoplastic therapy may also be responsible for inferior ccancer-related outcomes. The COVID-19 pandemic has, in fact, been associated with increased treatment delays, ${ }^{16}$ partly caused by patients' fears of becoming infected by this virus when treated in an outpatient day care facility. ${ }^{11}$ It follows, then, that measures for reducing the risk of exposure, ensuring continuity in safe treatment, reassuring patients about their safety, and reducing their levels of anxiety are mandatory. A survey performed in 21 oncological centers in 10 of the most corona-affected countries demonstrated that preventive measures, similar to those we took, had been established in most of those centers. ${ }^{17}$ However, the impact of such measures on patient anxiety and adherence to therapy has not been established.

According to our data, the employment of protective measures together with the adoption of continuing communication with the patients resulted in a significant decrease in patient anxiety level and equivalent compliance with the treatment schedule. As recently reported by others, anxiety levels were especially high in women $^{11,18,19}$ and in non-actively treated patients, probably reflecting the latter's lesser dependency upon medical services. Nevertheless, a remarkable decrease in anxiety levels was observed in all patients, an achievement which might also be due to the high proportion of the relatively highly educated patients in our cohort. Most patients, even those that were required to attend the outpatient clinic quite frequently, continued to receive their scheduled therapies without interruption, and most patients followed their planned blood tests and imaging study schedules.

Telemedicine in oncology has already been in use for many years, providing medical services for individuals that live in remote areas ${ }^{20}$ or are incarcerated. ${ }^{21}$ In the COVID19 era, telemedicine has become part of the armamentarium at the physician's disposal to decrease patient risk of contracting COVID-19, therefore dramatically increasing its utilization. Onesty et $\mathrm{al}^{17}$ reported that telemedicine was implemented in $76.2 \%$ of oncological centers that were screened in their study. Ramaswamy et $\mathrm{al}^{22}$ performed a retrospective observational cohort study (5\% were hematology/oncology patients) in which they analyzed patient satisfaction with video physician visits $(n=620)$ vs inperson physician visits $(37,989)$ at an academic medical center in New York City from April 1, 2019 to March 31,
2020. These authors reported an $8729 \%$ increase in video visit utilization during the COVID-19 pandemic compared to the same period of the previous year. Notably, video visit satisfaction scores were significantly higher than in-person visits $(94.9 \%$ vs $92.5 \%$; $<<0.001)$.

Although telemedicine may be welcomed by patients of higher socioeconomic levels (as demonstrated by $84 \%$ of our patients having a high school/college degree), it is imperative to identify patient populations who require greater resources to overcome access barriers to virtual care. In line with that, studies that evaluated telemedicine proposed that older and rural patients, as well as patients with lower median household incomes might face difficulties in using these high-tech technologies. ${ }^{23,24}$ Furthermore, Jazieh et al reported that medical centers in lower-income countries were less likely to hold virtual tumor boards and run virtual clinics. ${ }^{16}$

Interestingly, according to our analysis, satisfaction with the use of telemedicine was high in those patients experiencing high anxiety levels of attending the hospital, suggesting that telecommunication might be especially suitable for them. In contrast, the majority of patients that were actively treated, were not interested in continuing telemedicine, reflecting the relatively high frequency of treatment-related complications and a progressive nature of most hematological malignancies, promoting the employment "in hospital" medical services.

Our study has several limitations, mainly attributed to the small number and selection bias of patients that agreed to answer our survey. The study was designed and approved to capture the data of responders only. Therefore, we do not have detailed data regarding the entire patient population that visited our clinic/day care at the same time period. As a result, we recognize the possibility of a "non-response bias". This bias could explain the relatively high proportion $(84 \%)$ of patients who responded to the survey with an academic degree. In fact, the percentage of people with higher education is only $46 \%$ for the entire population that lives in the area and seeks medical service from our hospital (Israel Central Bureau of Statistics. 1995 Census of Population and Housing Jerusalem: 1998). This potential bias echoes prior publications, demonstrating an overrepresentation of highly educated individuals in survey research, involving voluntary participation. ${ }^{25}$

In addition, willingness to respond to our survey may reflect a bias that may impact the results of patient satisfaction surveys, leading to overestimation of the level of satisfaction. ${ }^{26}$ 
The findings of our study demonstrate that the adoption of precautionary measures in the hemato-oncology care setting can lead to a significant reduction in COVID-19related anxiety, enabling successful execution of the treatment plan and the keeping of scheduled appointments, ${ }^{27}$ both of which are crucial for optimal health outcomes and quality of life.

\section{Author Contributions}

N.S.- Conception or design of the work, Data collection, Data collection, Data analysis and interpretation, Drafting the article, Critical revision of the article, Final approval of the version to be published, He is a guarantor.

S.M.- Data collection, Data analysis and interpretation, Drafting the article, Critical revision of the article.

Y.H.- Data collection, Data analysis and interpretation,

Drafting the article, Critical revision of the article.

C.P.- Data collection, Data analysis and interpretation, Drafting the article, Critical revision of the article.

Y.C.- Data collection, Data analysis and interpretation,

Drafting the article, Critical revision of the article.

I.P.- Conception or design of the work, Data collection, Critical revision of the article.

M.N.- Data collection, Data analysis and interpretation, Drafting the article, Critical revision of the article.

N.B.- Data collection, Data analysis and interpretation, Drafting the article, Critical revision of the article.

M.J.- Conception or design of the work, Data collection, Critical revision of the article.

I.A.- Conception or design of the work, Data collection, Data analysis and interpretation, Drafting the article, Critical revision of the article, Final approval of the version to be published, She is a guarantor.

All authors made substantial contributions to conception and design, acquisition of data, or analysis and interpretation of data; took part in drafting the article or revising it critically for important intellectual content; agreed to submit to the current journal; gave final approval of the version to be published; and agree to be accountable for all aspects of the work.

\section{Disclosure}

The authors report no conflicts of interest in this work.

\section{References}

1. Guan WJ, Ni ZY, Hu Y, et al. Clinical characteristics of coronavirus disease 2019 in China. $N$ Engl J Med. 2020;382(18):1708-1720. doi:10.1056/NEJMoa2002032
2. Birenbaum-Carmeli D, Chassida J. Covid-19 in Israel: socio-demographic characteristics of first wave morbidity in Jewish and Arab communities. Int $J$ Equity Health. 2020;19(1):153. doi:10.1186/s12939-020-01269-2

3. Chen T, Wu D, Chen H, et al. Clinical characteristics of 113 deceased patients with coronavirus disease 2019: retrospective study. BMJ. 2020;368:m1091. doi:10.1136/bmj.m1091

4. Williamson EJ, Walker AJ, Bhaskaran K, et al. Factors associated with COVID-19-related death using OpenSAFELY. Nature. 2020;584 (7821):430-436. doi:10.1038/s41586-020-2521-4

5. Wu Z, McGoogan JM. Characteristics of and important lessons from the coronavirus disease 2019 (COVID-19) Outbreak in China: summary of a Report of 72314 cases from the Chinese center for disease control and prevention. JAMA. 2020;323(13):1239-1242. doi:10.10 01/jama.2020.2648

6. Isidori A, de Leval L, Gergis U, Musto P, Porcu P. Management of patients with hematologic malignancies during the COVID-19 pandemic: practical considerations and lessons to be learned. Front Oncol. 2020;10:1439. doi:10.3389/fonc.2020.01439

7. Lee LYW, Cazier JB, Starkey T, et al. COVID-19 prevalence and mortality in patients with cancer and the effect of primary tumour subtype and patient demographics: a prospective cohort study. Lancet Oncol. 2020;21(10):1309-1316. doi:10.1016/S14702045(20)30442-3

8. Mato AR, Roeker LE, Lamanna N, et al. Outcomes of COVID-19 in patients with CLL: a multicenter international experience. Blood. 2020;136(10):1134-1143. doi:10.1182/blood.2020006965

9. Passamonti F, Cattaneo C, Arcaini L, et al. Clinical characteristics and risk factors associated with COVID-19 severity in patients with haematological malignancies in Italy: a retrospective, multicentre, cohort study. Lancet Haematol. 2020;7(10):e737-e45. doi:10.1016/ S2352-3026(20)30251-9

10. Kurniawan A, Halim D, Sutandyo N. Multiple myeloma management in COVID-19 Era. Asian J Oncol. 2021;7(01):01-7. doi:10.1055/ s-0040-1716813

11. Karacin C, Bilgetekin I, F BB, Oksuzoglu OB. How does COVID-19 fear and anxiety affect chemotherapy adherence in patients with cancer. Future Oncol. 2020;16(29):2283-2293. doi:10.2217/fon2020-0592

12. Pareek P, Vishnoi JR, Kombathula SH, Vyas RK, Misra S. Teleoncology: the Youngest Pillar of Oncology. JCO Glob Oncol. 2020;6:1455-1460. doi:10.1200/GO.20.00295

13. Li Q, Chen L, Li Q, et al. Cancer increases risk of in-hospital death from COVID-19 in persons $<65$ years and those not in complete remission. Leukemia. 2020;34(9):2384-2391. doi:10.1038/s41375020-0986-7

14. Santosh KC. COVID-19 prediction models and unexploited data. J Med Syst. 2020;44(9):170. doi:10.1007/s10916-020-01645-z

15. Maringe C, Spicer J, Morris M, et al. The impact of the COVID-19 pandemic on cancer deaths due to delays in diagnosis in England, UK: a national, population-based, modelling study. Lancet Oncol. 2020;21(8):1023-1034. doi:10.1016/S1470-2045(20)30388-0

16. Jazieh AR, Akbulut H, Curigliano G, et al. Impact of the COVID-19 pandemic on cancer care: a Global Collaborative Study. JCO Glob Oncol. 2020;6:1428-1438. doi:10.1200/GO.20.00351

17. Onesti CE, Rugo HS, Generali D, et al. Oncological care organisation during COVID-19 outbreak. ESMO Open. 2020;5:4. doi:10.1136/ esmoopen-2020-000853

18. Ozdin S, Bayrak ozdin S. Levels and predictors of anxiety, depression and health anxiety during COVID-19 pandemic in Turkish society: the importance of gender. Int J Soc Psychiatry. 2020;66 (5):504-511. doi:10.1177/0020764020927051

19. Wang Y, Di Y, Ye J, Wei W. Study on the public psychological states and its related factors during the outbreak of coronavirus disease 2019 (COVID-19) in some regions of China. Psychol Health Med. 2020;1-10. 
20. Hamilton E, Van Veldhuizen E, Brown A, Brennan S, Sabesan S. Telehealth in radiation oncology at the Townsville Cancer Centre: service evaluation and patient satisfaction. Clin Transl Radiat Oncol. 2019;15:20-25. doi:10.1016/j.ctro.2018. 11.005

21. Young JD, Badowski ME. Telehealth: increasing access to high quality care by expanding the role of technology in correctional medicine. $J$ Clin Med. 2017;6(2):20. doi:10.3390/ jcm6020020

22. Ramaswamy A, Yu M, Drangsholt S, et al. Patient satisfaction with telemedicine during the COVID-19 Pandemic: retrospective Cohort Study. J Med Internet Res. 2020;22(9):e20786. doi:10.21 96/20786

23. Ferguson JM, Jacobs J, Yefimova M, Greene L, Heyworth L, Zulman DM. Virtual care expansion in the veterans health administration during the COVID-19 Pandemic: clinical services and patient characteristics associated with utilization. $J$ Am Med Inform Assoc. 2020.
24. Tam S, Wu VF, Williams AM, et al. Disparities in the uptake of telemedicine during the COVID-19 surge in a multidisciplinary head and neck cancer population by patient demographic characteristics and socioeconomic status. JAMA Otolaryngol Head Neck Surg. 2020. doi:10.1001/jamaoto.2019.3751

25. Cheung KL, Ten KPM, Smit C, de Vries H, Pieterse ME. The impact of non-response bias due to sampling in public health studies: a comparison of voluntary versus mandatory recruitment in a Dutch national survey on adolescent health. BMC Public Health. 2017;17 (1):276. doi:10.1186/s12889-017-4189-8

26. Mazor KM, Clauser BE, Field T, Yood RA, Gurwitz JH. A demonstration of the impact of response bias on the results of patient satisfaction surveys. Health Serv Res. 2002;37(5):1403-1417. doi:10.1111/1475-6773.11194

27. Puts MTE, Tu HA, Tourangeau A, et al. Factors influencing adherence to cancer treatment in older adults with cancer: a systematic review. Ann Oncol. 2014;25(3):564-577.

\section{Publish your work in this journal}

Patient Preference and Adherence is an international, peer-reviewed, open access journal that focusing on the growing importance of patient preference and adherence throughout the therapeutic continuum. Patient satisfaction, acceptability, quality of life, compliance, persistence and their role in developing new therapeutic modalities and compounds to optimize clinical outcomes for existing disease states are major areas of interest for the journal. This journal has been accepted for indexing on PubMed Central. The manuscript management system is completely online and includes a very quick and fair peer-review system, which is all easy to use. Visit http:// www.dovepress.com/testimonials.php to read real quotes from published authors. 